\title{
Kann denn Minne Sünde sein? Poetische Gratwanderungen zwischen Marien- und Frauenverehrung bei Hugo von Montfort und Oswald von Wolkenstein
}

\section{Vorbemerkungen}

Meine Überlegungen gelten in erster Linie der Marienlyrik Oswalds von Wolkenstein und nur am Ende auch zwei Texten Hugos von Montfort. Weiter einschränkend, greife ich aus dem ebenso reichen wie umstrittenen Euvre von Oswalds Marienliedern ${ }^{1}$ nur jene heraus, die einen so genannten Kippeffekt enthalten: Damit meine ich ein hermeneutisches Umschlagen in einem Text, der viele Verse lang als rein profane Verherrlichung einer Minnedame verstanden werden kann, der aber dann - auffallend spät - durch ein besonderes Dechiffrierungssignal klar macht, dass hinter oder neben der Gepriesenen die Gottesmutter Maria gemeint ist. Um diese Kippeffekte in weiterer Folge als poetisch geplante Wendepunkte diskutieren zu können, gilt es schon vorweg kritisch nachzufragen, ob sie auch in Anbetracht von historischer Melodieausstattung und inszenatorischer Umsetzung funktionieren konnten: Ich meine, ja, denn gegen die Annahme einer apriorischen Disambiguierung spricht, dass die relevanten Tongebilde Oswalds offen bleiben für eine sowohl profane als auch sakrale Hörart, und was den Inszenierungskontext angeht, müssen wir (überspitzt formuliert) als prä-monosemierende Aufführungsumstände nicht unbedingt an einen Marienfesttag denken mitsamt einem kuttentragenden Sänger in einer Kapelle, den Blick fest auf eine Marienstatue geheftet; dies hätte in der Tat jede noch so kunstvoll angelegte Textspannung schon im Keim erstickt.

1 Vgl. folgende rezente Publikationen zur Gattung Marienlied bei Oswald von Wolkenstein: Hartmann, S. 71-92; Spicker, S. 96-102; Löser, S. 251-261, besonders S. 253-255. Einen allgemeineren Blick auf dieses Thema findet sich schon davor - unter Einbeziehung Oswalds - bei Tervooren, S. 15-47. 


\section{Elementares zur literarhistorischen Marienverehrung}

Minnesang und Mariendichtung sind eng miteinander verflochten, schon ob ihrer genetischen Verbindungen; ihre Topik, Erlösungskonzepte und nicht zuletzt das über Jahrhunderte imaginierte weibliche Schönheitskonzept ergänzen einander, wirken mitunter sogar austauschbar. Um 1400 - also zur Schaffenszeit der beiden Dichter - hatte man sich sowohl in der Dichtung als auch in der bildenden Kunst ein besonders schönes, liebliches Bild von Maria gemacht. ${ }^{2}$ Welchen europaweiten Hype die Marienfrömmigkeit um die Zeit Hugos und Oswalds ausgelöst hatte, verdeutlicht etwa Klaus Schreiners Monographie, die zwischen Kulturgeschichte, Volksfrömmigkeit, Pastorallehre und Theologie das Leben und die Rezeption dieser bis heute weltweit verehrten Frau nachzeichnet. ${ }^{3}$ Vorausgegangen waren dieser Entwicklung u.a. die biblische Bildsprache im ,Hohen Lied der Liebe', apokryphe Quellen, die Bildtradition der Ostkirche, die westliche Mystik und damit zusammenhängend die Protomoderne der Gotik mit ihrer Ausformung einer emotionalen, ans überirdische Licht drängenden und den einzelnen Menschen mitreißenden Körperlichkeit. Projizieren wir diese inner- wie äußerlich emporstrebende Bewegung auf die krisenhafte Zeit um 1400, als Seuchenplagen, klimatische Veränderungen und Glaubenszerwürfnisse weite Teile Zentraleuropas, insbesondere dessen Süden stark verunsichert hatten, so wird verständlich, wie und warum die Modellierung Marias als Kryptogöttin ${ }^{4}$ dazu diente, zugleich kollektive und individuelle Erlösungssehnsüchte zu befriedigen. Daraus erhellt etwa das Phänomen der Schutzmantelmadonna als eine existentielle Heilsallegorie, die nicht zufällig um 1400 ihr inhärentes Schutz- und Erlösungsversprechen weithin zu entfalten begann, gegründet auf dem Imaginat von weiblicher Erhörungs- und Hingabebereitschaft bei gleichzeitiger leibgeistiger Unversehrtheit. ${ }^{5}$

2 Vgl. Knapp, S. 181-194.

3 Vgl. Schreiner.

4 Vgl. dazu Söll, S. 93-231. Aus dem Kapitel „3. Die Entfaltung der Marienlehre und Marienverehrung im Mittelalter“ stammt folgendes Zitat auf S. 168: „Albert der Große (1280) hat als erster bewußt die Mariologie der Christologie zugeordnet. Er lehnte die apokryphen Schriften als ,frivol ab, vertritt die Hyperdulia für die Gottesmutter, lehnt den Begriff der Anbetung für sie entschieden ab und spricht das Erlösungswerk ausschließlich Christus zu [...]“.

5 Dazu erläutert Schiller, S.196b: „In Deutschland und Frankreich breitet sich ebenso wie in Italien vom 14.Jh. an in Skulptur und Malerei der Typus der stehenden Schutzmantelmadonna aus und erreicht seinen Höhepunkt um 1500. In Italien ist von der gleichen Zeit an auch die thronende Madonna in dem dort üblichen Typus mit dem Schutzmantel zu finden. Nördlich der Alpen variiert die Marienfigur; die jungfräuliche Himmelskönigin herrscht vor.“ 


\section{Marientheologisches}

Die moderne Marientheologie weiß um die diskussionsintensive langwierige Entwicklung der Mariendogmatik, insbesondere um die starke Prägung der mittelalterlichen Marienverehrung durch Bernhard von Clairvaux. ${ }^{6}$ Daraus sei hier nur das Konzept der unbefleckten Empfängnis (immaculata conceptio) hervorgehoben, da diese für das mutmaßliche poetische Kippspiel zwischen einer irdisch berührbaren und einer überirdisch unantastbaren Frauenfiguration besonders relevant erscheint; dazu schreibt Georg Söll im ,Handbuch der Marienkunde':

Die Wortführerin unter den europäischen Universitäten, die Sorbonne in Paris, duldete zunächst beide Meinungen, da dort ja auch Vertreter beider Bettelorden dozierten. Die Toleranz der Alma Mater wurde aber bald auf eine harte Probe gestellt. Denn der spanische Dominikaner Johannes von Montesinos (1412) behauptete in aggressiver Form gegen Skotus, es sei ,gegen den Glauben', anzunehmen, daß Maria neben Christus als erbsündenfrei bezeichnet wird. Die Theologische Fakultät der Sorbonne ließ die Thesen durch den Franziskaner Johannes Vitalis prüfen und verurteilte am 6. Juli 1387 gemäß dem Gutachten von dreißig Professoren die Thesen als ,falsch, ärgerniserregend und voreilig ${ }^{6}[. .$.$] Rom hat sich in der$ Immaculata-Frage ungewöhnlich lange zurückgehalten und vor dem 15. Jhdt. keine Entscheidung getroffen. Einige Päpste vertraten vor ihrer Erhebung zum obersten Hirtenamt die Meinung der großen Scholastiker, doch nahm keiner nachher amtlich gegen die Immaculata Conceptio Stellung. ${ }^{7}$

In diese Debatte appellativ mit involviert wurde u. a. Kaiser Sigismund und damit wohl auch sein ,Diplomat und Botschafter' Oswald von Wolkenstein. 1439 befand

6 Dazu schreibt Söll, S. 168: „An Breitenwirkung zugunsten der volkstümlichen Marienverehrung kam wohl kein anderer Vertreter des Frühmittelalters Bernhard gleich. In seiner Epoche wurde der Titel Mittlerin für Maria im Abendland heimisch. Im Überschwang der Gefühle wurde die Grenze zwischen dem einen Mittler Christus und der ,Mittlerin zum Mittler` zuweilen verwischt, wenn der Mutter die Fähigkeit zugesprochen wird, selbst Verdammte zu retten und gleichsam durch eine Hintertür ins Himmelreich zu bringen, ein Gedanke, der den Widerspruch nicht nur der Reformatoren auslösen mußte.“ Präzisierend liest man dazu bei Esser, S. 13f.: „Mit Bernhard beginnt ein Perspektivenwechsel im mariologischen Denken: Vor dem Hintergrund des streng hierarchischen Denkens, das dem Mittelalter eigen war, bekommt auch Maria eine neue Rolle zugewiesen: Sie steht nicht mehr in, sondern über der Kirche [...] Die Mittlerschaft bedeutet für Bernhard auch, dass Maria ,der Weg' ist, auf dem Christus in diese Welt gekommen ist. Und in der ihr zugesprochenen Rolle als ,Fürsprecherin` tritt sie für die Schwächeren ein, wird so auch zur ,Anwältin‘ für die Sünder.“

7 Söll, S. 176. 
ein Konzilsbeschluss, dass Maria niemals von der Erbsünde befleckt wurde, ${ }^{8}$ doch die sogenannten Makulisten gaben nicht auf: „Der Dominikaner Vinzenz Bandelli veröffentlichte 1475 in Mailand ein Buch, in dem er über 200 Väterzeugnisse gegen die Immaculata Conceptio präsentierte und den immakulistischen Standpunkt als irrig, gottlos und gefährlicher denn die Häresie des Pelagius brandmarkte. “9 Dem trat man 1477 mit einem Immakulata-Hochfest in Rom entgegen. Zum Dogma wurde dieser Glaubensinhalt erst am 8. Dezember 1854, ein Feiertag ist ihm in den romnahen ,Glaubenslanden‘ Italien und Österreich geweiht, weiter nördlich jedoch nicht mehr. ${ }^{10}$

\section{Oswald von Wolkenstein}

Die tiefe Religiosität des Südtirolers Oswald von Wolkenstein bedarf hier keiner Erläuterung, auch nicht sein zähes, oft zerknirschtes Ringen um ein gottgefälliges Leben - Werk und Urkunden ${ }^{11}$ sprechen für sich. Erwähnenswert scheint jedoch, dass er sich innerhalb der vorhin erwähnten Marien-Reinheitsdebatte positionierte. Das verdeutliche die folgende Sammlung (in der sich das Narrativ von der unbefleckten Empfängnis oft mit dem von der jungfräulichen Gottesgeburt verschmolzen zeigt):

Ein edle junkfrou klar, / die zwar fürwar ein sun gebar, / der keuschlich ain ir vatter was;/ mäglichen rain si des genas, (Kl.13/7-10), die uns gebar / ein sun keuschlich zu freuden (Kl. 34/ 21f.), die in keuschlichen hie gebar / an we und mail, das sag ich eu für war. (Kl.111/17f.), haiss wainen si da übt / umb iren seligen, lieben schatz, / den si empfieng, gepar durch keuschen latz.

8 Vgl. dazu ebd., S. 176 f.: „König Alfons von Spanien schrieb mehrmals an den Kaiser Sigismund mit der Bitte, die Sache der Immaculata durch eine allgemeine Kirchenversammlung entscheiden zu lassen. 1431 trat unter dem Pontifikat Eugen IV. in Basel ein Konzil zusammen und ließ durch den Kardinal Ludwig Aleman von Arles in allen Bibliotheken nach Zeugnissen für und wider die Lehre forschen. In Basel selbst standen die Dominikaner und Franziskaner einander gegenüber. Obwohl schließlich das Konzil durch die Verlegung nach Ferrara 1437 seine Legitimation verlor und nur noch ein Rest der Konzilsväter mit ihren Theologen zurückblieb, traf man eine Lehrentscheidung zugunsten der Unbefleckten Empfängnis Mariens.“

9 Ebd., S. 181.

10 Der 8. Dezember wurde erst ab 1708 für die gesamte katholische Kirche ein verbindlicher Marienfeiertag, als gesetzlicher Feiertag gilt das Hochfest Mariä Empfängnis heute im europäischen Raum (nur) noch in Italien, Österreich, Liechtenstein, Portugal, Malta und in Teilen der Schweiz.

11 Vgl. dazu die monumentale Ausgabe von Anton Schwob und Ute Monika Schwob. 
(Kl.111/106-108), des si genas, / magt vor und nach, von ainer sprach, ,ave', an mail empfieng, gebar. $(\mathrm{Kl} .114 / 5-7)^{12}$

Schon dieser Zusammenschnitt drückt aus: Oswald war ein bekennender (!) Immakulist, und seine daraus geschöpfte Marienfigur verkörpert somit eine überirdisch reine, dem männlichen Körperbegehren entrückte Heilsbringerin. Das denken wir fort, wenn wir nun den Denotations-Kollisionen zwischen irdischer und himmlischer Liebesschau in Oswalds mariologischen Kipptexten nachspüren. Zuerst seien jene Lieder genannt, die unstrittig als Marienlieder gelten, auch wenn ihr Text dies erst markant spät zu erkennen gibt. Danach folgen jene ,Grenztexte', in denen - von der Forschung bislang nicht gesehen - wohl ebenfalls ein Umschlagen vom Profanen ins Sakrale stattfindet. ${ }^{13}$

Das Lied Kl. $34^{14}$ Es leucht durch grau ${ }^{15}$ funktioniert bis zur Hälfte, genauer bis vor die Zeile 21, tadellos als ein Tagelied mit einem betörenden Schönheitspreis

12 Diese und alle weiteren Textbeispiele zu Oswald von Wolkenstein sind der Referenzausgabe von Karl Kurt Klein (Hg.) entnommen. Ein älterer, bei unseren Liedern weitgehend wortgleicher Abdruck aller Texte ist online verfügbar: http://www.wolkenstein-gesellschaft.com/texte_oswald. php. Einen raschen vergleichenden Zugriff auf die gesamte handschriftliche Überlieferung erlaubt diese Seite: http://sosa2.uni-graz.at/sosa/nachlass/sammlungen/wolkenstein-archiv/OvW_Inter face/Handschriften_Interface.htm (14. Januar 2019).

13 Vorbemerkungen zur Textselektion: Mit Verweis auf die kurze Marienlieder-Bibliographie in Anm. 1 sei das Fehlen folgender Lieder in meiner Reihe der sogenannten Kipptexte kurz kommentiert: Kl. 13 ,kippt` nicht, sondern ist von Anfang bis Ende ein monosemantisch luzider Marienpreis. Gemeinhin nicht als Marienlied wird Kl. 33 gesehen, obwohl dessen sehr körperliches irdisches Liebesbegehren des einsamen Mannes auch an einen endzeitlichen Erlösungswunsch und damit an ein Geistliches Tagelied denken lassen könnte, allerdings ohne einen signifikanten ,Kippmarker‘ zu bieten (vgl. Hofmeister, S. 114 f.). In Kl. 36 finden sich meines Erachtens. keinerlei klare Marienbezüge. Kl. 38 hingegen ist von Beginn an als Marienpreis codiert und geht am Ende in einen Preis ihres Gottessohnes über, sofern man in V.73 den steren auf ihn, den Erlöser, bezieht; siehe dazu den Kommentar bei Hofmeister, S. 127. Ohne überraschend zu kippen, changiert K1. 78 durchgehend zwischen einem überirdischen und einem - dahinter mit angelegten - weltlichen Erlösungsbegehren, wobei nicht zuletzt die signifikante Stimmführung der Melodie die sakrale Lesart von Anfang an in den Vordergrund rückt (vgl. Spicker, S. 102f.). Ähnliches scheint musikalisch wie inhaltlich für das Lied Kl. 120 zu gelten, zumal dessen Eingangsvers Fräu dich, du weltlich creatur den Einstiegsvers der 2. Strophe von Kl. 78 wiederholt beziehungsweise zitiert; dazu schreibt Knapp, S. 194: „Wir dürfen es [= Lied Kl. 120] also der geistlichen Sphäre zuschlagen. Das Gespür für solche Nuancen wollte Oswald seinem Publikum offenbar zumuten. Bei aller menschlichen Nähe des männlichen Verehrers zur seligen Jungfrau blieb diese in ihrer immerwährenden Keuschheit erotischem Begehren entrückt.“

14 Zur Einordnung dieses Liedes in den Marienlieder-Kanon vgl. Wachinger, S. 110: „KL 34 ist also ein Marienlied mit Tagesanbruchs- und Weckmotiven. “ Eine bewusste Bezugnahme Oswalds von Wolkenstein auf die Madonnen-Malerei des Jan van Eyck in Kl. 34 erwägt Hartmann, S. 84-86. 
der betrachteten Geliebten. Doch die monosemantische Hinwendung an die kron, die uns gebar / ein sun keuschlich zu freuden (Kl. 34/21f.) vertreibt diese irdische Fata Morgana und macht den Text zur Todesvision eines Erlösung suchenden lyrischen Ichs. ${ }^{16}$ Metapoetisch gewendet, könnte man hier hintersinnig verstehen: Miterlöst, weil bestätigt könnten sich all jene im Publikum sehen, die frühzeitig hinter der poetischen Topik ein latentes Oszillieren zwischen Dies- und Jenseits wahrgenommen, also eine doppelte Codiertheit bereits geahnt hatten. Sie haben von der Zweispurigkeit auf dem irdischen Weg Richtung Erlösung oder Verderben gewusst, die Abzweigung in Richtung des Heilswegs hat sie nicht überrascht; anders als die verdutzten und nun beschämten Männer und Frauen dürfen sie sich als gut vorbereitete Textpilger am überirdischen Zielort willkommen wähnen. - So oder ähnlich könnte man den rezeptiven Effekt von Oswalds doppeldeutigem Grenzgang auch für die nächsten Kipptexte formulieren.

Anfangs ebenso sicher nur an eine Frau aus Fleisch und Blut gerichtet wirkt das naturverbundene ,Erweckungslied“ Kl. $37^{17}$ (Des himels trone), zumal es in Zeile $25 \mathrm{f}$. eine liebevolle Umarmung imaginiert: freuntlich schrenke, / die sie kan. Doch sobald die Zeilen 57-60 weiplich zucht, / gedenk an mich, / wenn ich / kom zu dir an den tanz die Frau erstmals adressieren, kann man diese Erlösungsbitte kaum mehr anders verstehen denn als eine Anrede an die Jungfrau Maria, und die hilfesuchende Bitte um ihr ,Eingedenksein beim bevorstehenden Tanz' evoziert daraufhin den post-eschatologischen Auferstehungsreigen. Retrospektiv erfährt Des himels trone (Kl. 37,1) eine disambiguierende Recodierung in Richtung Marienverehrung, und aus der scheinbar rein höfisch vorzustellenden frouen (Kl. 37/12) des Sängers ist die überirdische ,Herrin' geworden. ${ }^{18}$ Überraschend nur für alle hermeneutisch Verschlafenen im Publikum, endet das Tagelied nach seinem ebenfalls transzendent auslesbaren - Naturerneuerungspreis in Strophe III mit einem Gotteslob und Gnadendank.

15 Zur hier (weiter) verwendeten Referenzausgabe vgl. Anm. 12.

16 Genau diese Stelle in Kl. 34 hat schon Knapp, S. 191 als eine Art von disambiguierendem Kipppunkt gesehen: „In Kl. 34 hat der Dichter ein weltliches Tagelied nur geringfügig auf ein Marienlob umgebogen, so daß nur einzelne Verse wie 21f. die Adressatin preisgeben. Die schöne, raine maget, zart junkfrau, rain creatur von unvergleichlicher Schönheit, an tadels mail, wird sogar mit einem veinen mündlin rot (35) ausgestattet.“

17 Löser, S. 254 kategorisiert Kl. 37 gemeinsam mit Kl. 38 (in expliziter Anlehnung an die Marienliedereinteilung von Hartmann, wie Anm. 1) als „zwei Loblieder in Form von höfischen Tanzliedern“.

18 Vgl. Hofmeister, S. 122 mit Bezugnahme auf die Übersetzung von frouen als „Herrin“: „, (auch) die Gottesmutter Maria?! (Vgl. dazu die spirituelle Mai-Metaphorik in Kl. 40)“. 
Das weitgereiste lyrische Ich in $\mathrm{Kl} .12^{19}$ In Frankereich bejubelt seine Geliebte durch eine Steigerung der klassischen Minnesang-Überbietungstopik mittels autobiographisch eingefärbter Bewahrheitungen als die Schönste aus zumindest 16 Ländern und wünscht sich $\mathrm{zu}$ ihr in iern garten (33) mit den rosen (34) und ersehnt sich dort von ihr nicht mehr, aber auch nicht weniger als ein (topisch hoch aufgeladenes) krenzlin grüen (35): Das würde er ihr gerne ab erkosen (32). Glücklich alle Zuhörenden, die schon da auch oder nur mehr an Maria dachten.Nach diesem symbolreichen Ausflug in den Garten der Freuden gelten die Gedanken des lyrischen Ichs wieder seinen eigenen Reiseerinnerungen. Erst in Strophe IV folgen mit Blick auf die Geliebte neuerliche, jetzt noch prunkhaftere Unerreichbarkeitsbilder, die jedoch - so hören wir - allesamt verblassen müssten vor dem einen allergrößten schatz (62), den die Zeilen 67-70 schildern: An tadel, rain, diemüetiklich, / mit aller tugenthait, / in allem wandel züchtiklich, / so herscht die schöne maid. Solch inthronisiertes ,Herrschen', zumal ein Herrschen über alle und nicht bloß über den Sänger, disambiguiert die beiden bis hierher noch doppelläufig plausiblen Sinnlinien und lässt den Text ins Mariologische kippen. Dort macht ihn hernach der Schlusssatz der Strophe fest: umb trauren gäb ich nicht ein stro, / wil si mir wol, so fürcht ich niemands dro. (71f.) Die anschließend in der Strophe V genannte frouen schar (73), der der Sänger nicht mehr zu dienen gedenke, wird mit einem interjektiven Ach (73) adressiert: Vor dem nunmehr präsenten Hintergrund der Weltfluchtmotivik klingt dieser Seufzer wohl eher geringschätzig, verächtlich zurückweisend wie an ,Frau Welt‘ gerichtet. Zwar wird Maria als deren Gegenspielerin nicht deutlicher genannt, doch wer wüsste denn sonst beziehungsweise besser als sie seit jeher, ihr Ansehen so unversehrt zu wahren und persönliche Treue verlässlich zu entgelten? - Oswald hat diesem Text die Melodie seines vorangegangenen Weltfluchtliedes $O$ snöde werlt (Kl. 11) zugeordnet und damit allen Hellhörigen die Polydimensionalität seines Textgebäudes zwischen einem Hier und Dort, zwischen Frauen- und Marienverehrung

19 Vgl. dazu die Ausführungen bei Schulze-Belli, die sich auf S. 283-293 auf Kl. 12 konzentriert und vor allem Parallelen aus der mystischen deutschsprachigen Dichtung für die Verschmelzung von höfischen und mariologischen Dimensionen ins Treffen führt. „Das autobiographische Marienlied Kl. 12“ lautet eine vielsagende Kapitelüberschrift bei Hartmann, S. 81, die im Schönheitspreis der Strophe II die poetische Spiegelung eines französischen Schutzmantelmadonnenbildes aus der Zeit des Konstanzer Konzils erkennt. Zur umfangreichen Argumentation, wonach im Bildnis unter Marias Mantel auch Oswald von Wolkenstein zu sehen sei, vgl. Hartmann, Bildzeugnis, S. 297-332. Zur ,Marienhaltigkeit‘ von Kl. 12 merkt Knapp, S. 191f. an: „In Kl. 12 und Kl. 78 geht die Durchdringung von geistlicher und weltlicher Lyrik so weit, daß die Zuordnung zu einer der beiden umstritten ist.“. 
signalisiert, aber noch nicht den spannungsgeladenen Zeitpunkt des textuellen Kippens vom Dies- ins Jenseits verraten.

Folgt man den Überlegungen von Frank Fürbeth, so kippt nach vier Strophen auch das latent doppelzüngige Erlebnislied Kl. 18 endgültig in die Marienfrömmigkeit:

Und diejenige, die den Erzähler zu seiner Beichte erst zwingt und damit aus dem Zustand des Weltverhaftetseins befreit, ist sicherlich nicht die Minnedame, die mit ihrer minn die Konversion gestört hat und somit den Erzähler erst recht wieder in die Welt gezogen hat, und es ist sicherlich auch nicht eine jener Damen, die mit ihrer Minne nur laid bereiten: Es ist die höchste aller Minnedamen, die Muttergottes selber. ${ }^{20}$

Dadurch wird nachträglich - so ergänze ich - schon die Kutten- beziehungsweise Pilger-Maskerade des lyrischen Ichs in Strophe III mit dem Blick auf ein ausserweltes mündli rot / da von mein herz ist wunt bis in den bittern tod (66f.) zu einer Vorbotin der inhärenten Gottes- respektive Mariensuche. - Ähnlich wie in Kl. 12 bleibt diese mariologische Deutung bis zum Lied-Ende stimmig, bietet aber keine noch klarere Disambiguierung in Richtung Maria. Expliziter als in Kl. 12 wird am Textende von Kl. 18 jedoch die Jenseitsschau im Wissen ums eigene Sterben bei gleichzeitiger Unkenntnis des genauen Zeitpunkts dieses ,Kippens‘ ins Jenseits: Und wol bekenn, ich wais nicht, wenn ich sterben sol (109).

Ich komme nun zu drei Texten Oswalds, deren Mariologizität sich meines Wissens bislang nur in der Neuauflage meiner Gesamtübersetzung ${ }^{21}$ von Oswalds Liedern angedeutet findet: In Kl. 118 Wol auf und wacht - einem geistlichen Tagelied $^{22}$ - mahnt der Wolkenstainer (46) persönlich alle, die verschlafen haben, Mann wie Frau, rechtzeitig aufzuwachen. Eine retrospektive mariologische $\mathrm{Zu}$ spitzung erfahren die ersten beiden Strophen am Ende von Strophe II, wo von einem sich laben / mit der höchsten pluemen lobesan (25f.) die Rede ist, also gemäß diesem Überbietungstopos von Maria. ${ }^{23}$ In weiterer Folge rekurriert der Text indes nur noch auf den Erlösungstod Christi und die rettende Trinität; Maria scheint da allenfalls noch mitgedacht.

Etwas vager muten die Marienbezüge in den Liedern Kl. 81 und Kl. 107 an, doch tritt in ihnen auf sehr signifikante Weise das Wort weib als ein semantisch disambiguierender Kipp-Indikator in Erscheinung: Der Text Kl. 81 Sweig guet gesell, schimpflichen lach wirkt auf den ersten Blick nur wie eine launige Le-

20 Fürbeth, S. 213.

21 Hofmeister (wie Anm. 13).

22 Vgl. Spicker, S. 89.

23 Die hermeneutische Lösungsformel dazu hatte in der Neuauflage meiner Übersetzung (S. 308) - noch etwas unsicher - „= Maria?!“ gelautet. 
benslehre, er geht aber in der Schlussstrophe durch das Überlob für ein zart schön weib, mit er behuet (29) in einen Marienpreis über, denn, dass mit diesem auffallend ,reinen' Tugendlob wohl die Maria gemeint ist, bestätigen zum einen die gleichzeitige Zurechtweisung der weltgewandten freulin rain (17) und zum andern die Schlussverse des Liedes mit dem schwelgerischen Dank an den Schöpfergott dafür, dass er in vollkommener Harmonie ${ }^{24}$ ain schön weib tadels frei / schon würkt, der lob für alles gold / erleucht (32f.).

Selbst diejenigen, die meine Übersetzung des schäkernden Eheliebesliedes Kl. 107 Kom, liebster man schon kennen, mag meine jetzt neue Beurteilung als ein verkapptes beziehungsweise gekipptes Marienlied überraschen. Vormals hatte ich das nämlich zwar wortreich erwogen, doch zugleich verworfen. ${ }^{25}$ Nun bewerte ich meinen einstigen Hinweis auf das in Zeile 16 (wie in Kl. 81 vorhin) merkwürdig zäsurierende ain stolz weib jedoch noch stärker als eine valide Marien-Chiffre: Die eine, irdische Frau hatte - so sei das Lied nun gedeutet - dem Sänger körperliche Liebesfreuden geschenkt, die andere verheißt ihm die innere Erlösung. In dieses Verstehenskonzept passt die nachfolgende Jungbrunnen-Motivik (die mir das herz an smerz / verjüngt mit liebem scherz, 17f.) mitsamt den klaren öuglin, durch die sich das lyrische Ich benetzt (22) und erlösbar wähnt. Was hier hinter der sublimen Abschieds-Metaphorik widerhallen könnte, ist die Marienlied-typische eschatologische Dimension. Einmal auf dieser Deutungsebene angekommen, gehört die tränenreich mitfühlende Frauenstimme der letzten Strophe III ganz allein Maria, und ihre schutzbeflissene Sorge um den Lebenswandel und die sichere Heimkehr ihres Verehrers emaniert zu einer mystischen Marienbegegnung. - Von hier aus sei anhand des Stich-, ja Code-Worts weib wenigstens ein Seitenblick auf das Tagelied Kl. 40 Erwach an schrick, vil schönes weib geworfen, für das Burkhard Wachinger festhielt: „eine weltliche Deutung geht bei ihm nicht auf“. ${ }^{26}$ Tatsäch-

24 Übersetzung der gesamten Passage (Kl. 81/31-35): „Auch bin ich Gott von Herzen zugetan, weil er eine so schöne Frau, ganz ohne Makel, herrlich erschaffen hat, deren Ruhm alles Gold überstrahlt und die Tugendhaftigkeit sowie Ansehen ganz augenscheinlich harmonisch verkörpert!“ (Hofmeister, S. 216); die Anm. dazu lautet: „,an offenbars verstreuen wörtlich ,offensichtlich unverstreut', also (abstrakt verstanden), widerspruchsfrei ${ }^{\text {‘ }}=$, harmonisch'. Oder ist hier ,ohne viel öffentliches Gerede‘ gemeint? Damit würde sich aber das (hinter dem weib etablierte) Bild der Gottesmutter nur bedingt übereinstimmen lassen, da diesem sehr wohl und prinzipiell öffentliche Aufmerksamkeit zustehen würde.“ (Hofmeister, S. 216).

25 So hatte ich bei der Übersetzung von seid mein begerd / ain stolz weib junk hoch und werd (Kl. 107/15f.) durch den Wortlaut ,dass mich eine derart vornehme, junge, edle und ehrenhafte Frau begehrt“ (mit dem darin frei hinzugefügten Adverb „derart“) den Textsinn an die davor erwähnte Partnerin rückgebunden, aber selbstkritisch angemerkt: „Ohne dieses Adverb bliebe die Stelle offen für eine andere (Richtung Gottesmutter weisende??) Beziehung.“ (Hofmeister, S. 268). 26 Wachinger, S. 108. 
lich wirkt die Chiffre vil schönes weib schon zu Beginn wie eine Losung für Maria und bündelt stimmig die um die Gottesmutter und den Kreuzestod Jesu kunstvoll mehrdeutig gerankte Auferstehungsthematik. ${ }^{27}$ Es scheint somit durch das von Anfang bis Ende in Schwebe gehaltene Mit-Verstehen der überirdischen Erlösungsvision hinter der weltlichen Naturfassade etwas Marienliedhaftes mit angelegt zu sein, jedoch nicht das uns hier bewegende Kippen von Letzterem in Ersteres.

Als nur scheinbar blasphemischen Höhepunkt meiner eigenen Gratwanderung zwischen Minne- und Marienkult bei Oswald von Wolkenstein lade ich noch dazu ein, den überschwänglich detailreichen Schönheitspreis Kl. 87 ebenfalls als einen verkappt gekippten Marientext auszulesen: Mit eren, ausserweltes M., / liebst du mir in meins herzen grund (33f.), heißt es dort in der letzten Strophe, was in der Forschung beharrlich bloß auf Oswalds Ehefrau Margarete von Schwangau bezogen wird. ${ }^{28}$ Geht man jedoch von der häufigen mittelalterlichen Kürzungspraxis für ,Maria‘ aus, scheint mein ahnungsschwangerer Übersetzungskommentar zu dieser Abbreviatur gerechtfertigt: „M passt ebenso zu ,Margarethe ${ }^{\text {‘ wie }}$ zu ,Maria‘،"29 Und wenn wir noch einen Schritt weiterdenken, so werden wir förmlich gezwungen, bei dieser Chiffre nur mehr an die biblische Erlöserin zu denken, denn lediglich Oswalds Intimi konnten (so wie wir Forschenden heute wieder) hinter dem $M$. eine Anspielung auf seine Ehefrau erkennen. Hätte dieser Buchstabe einzig Oswalds Frau bezeichnen sollen und nicht auch ambig für ,Maria‘ stehen dürfen, wäre Oswald sein eindeutigeres ausserweltes $G$ zu Gebote gestanden, also jene Kürzung, wie er sie in Lied Kl. 68/11 als die Koseform für ,Gret‘ verwendet und zugleich dechiffriert hat. Falls wir mithin wirklich einmal mehr in Marien Schoß gelandet sind beziehungsweise - anderes Bild - uns gleichsam unter ihrem Schutzmantel wiederfinden, lassen wir uns auch nicht von der Melodiezuweisung an das frivol schillernde jetterin-Lied in Kl. 83 irritieren, sondern deuten unsere fröhliche Repeticio bußfertig um, indem wir beim finalen Zuruf an die aller liebste frau (Kl. 87/16) auch - oder gar nur mehr - an Maria denken. Doch zugegeben: Spätestens hier wird Interpretation zur Glaubensfrage.

27 Vgl. die schlüssige Deutung dieser Polyvalenz bei Schiendorfer, S. 179-196.

28 Vgl. Spicker, S. 66 sowie Knapp, S. $188 \mathrm{f}$.

29 Hofmeister, S. 229. 


\section{Hugo von Montfort}

Der alemannisch-steirische Graf und ,Gelegenheitsdichter Hugo von Montfort hat sich in seinem stark glaubenszentrierten Liedschaffen ebenfalls als Immakulist und mariologischer Virginitätsverteidiger positioniert, wie aus dieser Ährenlese deutlichst hervorgeht:

sy was din múter und doch ain magt / án all mail - daz glob ich wól. (Nr. 4, 100f.), min gott ist von ainer magt geborn. (Nr. 5, 107) des hilf mír magt an alle maíl, / das ich werd miner súnden hail! (Nr. 10, 34f.) behút mich vor der helle nót / durch deinen herten willig tót, / den du doch hast erlitten, / und durch die maget, die dich gebar! (Nr. 12, 11-14), got vatter, herr, durch all dein gnad / und durch die magt, die múter dein (Nr.18, 161 f.), herr gott, erbarm dich úber mich ellenden / durch die magt, die dich gebar (Nr. 28, 669f.), múter und magt, ain ros der tugent, / ain schilt der súndér, / ich man dich an deins kindes iugent /und als du in gebér, / pitt fúr mich, du raine magt / (er tút dir nichts verzeihen)! (Nr. 27, 165-170) das widerbracht die magt, / die iunkfraw, múter raín (Nr. 32/77 f.), zwelff sternen auff dem tach / sint liepleich an ze schowen, / als sant Iohans da sach / ain figur únser frawen. / die maget rain trait si ze kron /(án alle mail ir kewscher leib), / des neýgent ir die engel schon; / si ist ain magt, genant ain weib. (Nr. 28, 569576), das widerbracht die magt, / die iunkfraw, múter raín (Nr. 32, 77 f.), das kind ist geborn / von ainr iunkfrawen. (Nr. 32, 89f.). Belege aus dem (Hugo kongenial ,zugetrauten', doch kaum von ihm stammenden) Nachtragstext Nr. 39: [Rep.] ich meýn die súße María, / díe ýn gebar on smertzen. / sie trúg ýn nún und drýßig wochen da / under ýrem iungkfreulichem herrtzen. (Nr. 39, 20 - 23), du fraw, múter, iungfreuliche meýdt! (Nr. 39, 125), des hilff uns, María, reýne meýdt! (Nr. 39/130). ${ }^{30}$

Bei Hugo von Montfort findet sich jedoch kein einziges Lied, das - ähnlich wie bei Oswald von Wolkenstein wenig später so häufig - ins Mariologische kippt. Nur auf den ersten Blick scheint dieser Behauptung die Nr. 13 Ich var uff wag des bittern mer zu widersprechen, aber dieser Text enthält kein erst spät umschlagendes Frauen-Vexierbild, sondern man weiß sich von Beginn weg unterwegs auf einer allegorisch moralschweren Meerfahrt, die auf Überirdisches verweist: Das Schiff des Lebens sei nur von jener marnerin (15) richtig zu steuern, hinter der - schwach bis kaum als Anti-Eva maskiert - die Gottesmutter Maria erkennbar ist; sie ist es, die dann in den letzten beiden Zeilen ganz direkt den Bittruf des lyrischen Ichs entgegennimmt: o magt, ain lieb der drivaltikait, / bis bittens unverdrossen! (59f.).

Wer solche glaubensfesten Texte des Hugos von Montfort kannte, mochte auch in bzw. hinter seiner gottesfürchtigen Minnerede An dich gedenkhen hát erkikht (Nr. 1) eine Enthüllung der dort eingangs angesprochenen trut kayserin (4/

30 Diese und die weiteren Textstellen zu Hugo von Montfort sind der Ausgabe von Hofmeister entnommen. 
5) als Maria vermuten, speziell dann, wenn man dazu die topische Figur einer post mortem thronend entrückten Maria vor Augen hatte, etwa in jener Gestalt, die ihr Heinrich Aurhayms in seiner Miniatur zu diesem Text in der Heidelberger Hs. cpg 329 gab. ${ }^{31}$ Doch anstatt sich ins Überirdische zu erheben, senkt und vereindeutigt sich diese Rede zu einem hausväterlichen Verhaltenskodex für eine durchaus leibhaftig verehrte Frau: Ihre täuschend mariengleichen Züge brechen sich zumindest aus heutiger Sicht - spätestens in den Versen $24 \mathrm{f}$., die einen für die Gottesmutter kaum angemessenen Befehl äußern : zartú frow so tugentbár, / du volg meíner ler! Das Ende dieser Versrede offenbart schließlich eine unverhohlen begehrliche Unterwerfungsgeste des koketten Verehrers: du macht auch wol versúchen mich, wie dikh du wilt, meín hochster hort. (89f.). In der ,Colmarer Hs. von 1462/64 ${ }^{32}$, einer Sammelhandschrift mit rein erbaulich-belehrenden Texten, ist es (offenbar schon in historischer Zeit) zur Tilgung dieses Textes gekommen, nachdem - so vermute ich - der Revisor nachträglich erkannt hatte, dass der Thron der kayserin nicht von der Himmelskönigin besetzt wird, sondern von einer überhöhten Minnedame, deren Huldigung dem Sammelprogramm der glaubensfesten Handschrift zuwiderläuft. ${ }^{33}$ Für diese Vermutung eines ,sündhaften Rezeptionsirrtums' spricht noch heute die verräterisch eng benachbarte Federprobe mit dem erwartungssicheren Schriftzug Maria. ${ }^{34}$

\section{Conclusio}

Konnte Minne Sünde sein ${ }^{35}$ Auf Seiten des historischen Publikums mochte das in der Tat so empfunden worden sein, wenn man - gefangen in der Profanie eines

31 Vgl. diese (wohl 1414 entstandene) Darstellung auf fol. $1^{\mathrm{r}}$ unter https://digi.ub.uni-heidelberg. de/diglit/cpg329 (14. Januar 2019).

32 Vgl. die Angaben zur Hs. Colmar, Stadtbibl., Ms. 84, unter http://www.handschriftencensus. de/2545 sowie das Digitalisat unter https://bvmm.irht.cnrs.fr/consult/consult.php?COMPOSITI ON_ID=12868 (14. Januar 2019) (mit der Tilgung des mutmaßlichen Textes Nr. 1 Hugos von Montfort auf fol. $128^{\text {rv }}$ ).

33 Vgl. dazu die näheren Ausführungen in der Textausgabe (wie Anm. 30), S. XXVIf.

34 In zweifacher Ausformung direkt vor dem Tilgungsbeginn auf. fol. 128 ${ }^{\mathrm{r}}$ (wie Anm. 32).

35 Spätestens jetzt sei die Anspielung dieses - hier am Ende neuerlich aufgenommenen Aufsatzübertitels an Zarah Leanders Erfolgssong 'Kann denn Liebe Sünde sein' kurz expliziert (siehe den Songtext z.B. unter http://lyrics.wikia.com/wiki/Zarah_Leander:Kann_Denn_Liebe_ Sünde_Sein\%3F): Dieser Text lädt sein Publikum zu einer moralischen Gratwanderung ein, gebzw. verführt durch eine Femme fatale, man könnte auch sagen, durch eine begehrende und selbstbewusst begehrenswerte Geliebte im stets aktuellen Gewand der Frau Welt. Das daraus produktiv parallel entspringende Stichwort mag die, Weltvergessenheit‘ sein. 
rein erotischen Erwartungshorizonts - in den oben präsentierten Kipptexten zu spät deren Sinnangebot zwischen einem vorgeblich rein irdischen, doch in Wahrheit sublimiert verklärten Glücksbegehren erkannte und solcherart an der Muttergottes einen ,hermeneutischen Sündenfall' beging. Oswalds fast provokant irreführendes Oszillieren zwischen Profanie und Sakralität stellt sich als ein kongenialer Teil seiner gesamten abwandlungsfreudigen Poetik dar, als ein Spiel mit dem Minnefeuer ${ }^{36}$, das jedoch - um im Bild zu bleiben - nichts Geheiligtes verbrennen oder aus reiner Effekthascherei gar blasphemisch ,verbraten' wollte. Eher scheint es, dass Oswald für sein Publikum auf einigen seiner Textpfade zwischen Verderben und Erlösung ein nicht sofort sichtbares, doch genau dadurch umso eindrücklicher alles überstrahlendes Orientierungslicht entzünden und dieses auf die Gottesmutter Maria richten wollte. Dass hinter Oswalds Technik der semantischen Überblendung bzw. Disambiguierung freilich mehr steckt als eine Fortsetzung seiner bekannt virtuosen Mehrdeutigkeitsspiele, macht indirekt der Religionswissenschafter Thomas Lentes unter Bezugnahme auf den frühneuzeitlichen Philosophen Gianfrancesco erkennbar: Lentes spricht von einer „Kraft der Imagination, jener Seelenkraft, die die Brücke zwischen äußerer Wirklichkeit und innerem Menschen zu schließen vermochte“ und die in Gianfrancescos Traktat De imaginatione (1501) „gleichermaßen als Zusammenfassung der mittelalterlichen Imaginationstheorien wie auch eines neuzeitlichen Neuansatzes gilt“"37. Für uns darin aufschlussreich scheint nachfolgender kathartischer, auch die Marienfrömmigkeit einschließender Denkansatz zwischen Verführung und Heilssuche:

Die Therapie der Imagination erfolgt schließlich in allen Bereichen des Denkens, des gesellschaftlichen Handelns und der Religion über die Reinigung der Imagination. Die falschen und schadhaften Bilder sollen dabei wiederum durch andere, positivere Bilder ersetzt werden. ${ }^{38}$

Dies führt auf direktem Weg zurück zur bilderreichen, visuell verdichteten Marienverehrung unserer Texte und - mit Lentes weiterargumentiert - zur letztmaligen Frage, was genau darin sündhaft verfänglich sein konnte, nämlich schon allein

36 Wachinger, S. 114: „Solche erotische Rede von und zu Maria, wie Oswald sie hier wagt, hat, soviel ich sehe, in der deutschen Dichtung der Zeit kaum ihresgleichen. Dabei war die Verknüpfung von geistlicher Thematik und erotischer Bildlichkeit keineswegs ganz ungebräuchlich.“ 37 Lentes, S. 193. Der gesamte Band ist online verfügbar in der Digitalen Bibliothek des Münchener Digitalisierungszentrums (MDZ) unter https://digi20.digitale-sammlungen.de/de/fs1/ob ject/display/bsb00041583_00001.html (14. Januar 2019).

38 Lentes, S. 194. 
der Blick oder das innere Begehren? Dabei wurde in der Regel sehr genau differenziert, wann der Blick sündhaft ist und wann nicht. Stephan von Landskron [1412-1477] etwa trifft ganz in scholastischer Manier die Unterscheidung zwischen beabsichtigtem und unbeabsichtigtem Handeln. Der Blick allein genügt nach ihm noch nicht zur Sünde. Erst wenn der Blick von der Begierde begleitet wird, läßt sich von Sünde reden. ${ }^{39}$

Damit sollte am Ende noch klarer geworden sein, welch hintergründiger und feinsinniger Natur Oswalds von Wolkenstein poetische Gratwanderungen in seinen sogenannten Kipptexten waren: Fast traumwandlerisch zwischen Betrachten und Begreifen balancierend, scheinen sie Teil einer überirdischen Schule des heilsstiftenden Sehens zu sein.

\section{Literaturverzeichnis}

Esser, Günter: Zur Geschichte der Marienverehrung: ein Überblick. In: Internationale kirchliche Zeitschrift: neue Folge der Revue internationale de théologie 99 (2009), S. 4-32. Online unter: http://doi.org/10.5169/seals-405047 (22. Oktober 2019).

Fürbeth, Frank: wol vierzig jar leicht minner zwai im Zeichen der verkehrten Welt: Oswalds Es fügt sich (Kl 18) im Kontext mittelalterlicher Sündenlehre. In: Jahrbuch der Oswald von Wolkenstein-Gesellschaft 13 (2001/2002), S. 197-220.

Hartmann, Sieglinde: Ein neues Bildzeugnis Oswalds von Wolkenstein? Die Schutzmantelmadonna von Le Puy-en-Velay und das Marienlied In Frankereich. Mit einer kostümgeschichtlichen Untersuchung von Elisabeth Vavra. In: Jahrbuch der Oswald von Wolkenstein Gesellschaft 13 (2001/2002), S. 297-332.

Hartmann, Sieglinde: Gotische Madonnenbilder und die Marienlyrik Oswalds von Wolkenstein. In: wort und wîse, singen unde sagen. Festschrift für Ulrich Müller zum 65. Geburtstag. Hg. von Ingrid Bennewitz. Göppingen 2007 (Göppinger Arbeiten zur Germanistik 741), S. $71-92$.

Hofmeister, Wernfried: Oswald von Wolkenstein. Das poetische Werk. Gesamtübersetzung in neuhochdeutsche Prosa mit Übersetzungskommentaren und Textbibliographien. Berlin, New York 2011 (De Gruyter Texte).

Knapp, Fritz Peter: Das weibliche Schönheitsideal in den Liedern Oswalds von Wolkenstein. In: ZfdA 131 (2002), S. 181-194 (mit einem Abbildungsanhang).

Lentes, Thomas: Inneres Auge, äußerer Blick und heilige Schau. Ein Diskussionsbeitrag zur visuellen Praxis in Frömmigkeit und Moraldidaxe des späten Mittelalters. In: Frömmigkeit im Mittelalter. Politisch-soziale Kontexte, visuelle Praxis, körperliche Ausdrucksformen. Hg. von Klaus Schreiner. München 2002, S. 179-220.

Löser, Freimut: Oswald von Wolkenstein: Geistliche Lieder. In: Oswald von Wolkenstein. Leben - Werk - Rezeption. Hg. von Ulrich Müller, Margarete Springeth. Berlin, New York 2011 (De Gruyter Studium), S. 251-261, besonders S. 253-255.

39 Ebd., S. 203. 
Hugo von Montfort: Das poetische Werk. Hg. von Wernfried Hofmeister. Mit einem Melodie-Anhang von Agnes Grond. Berlin, New York 2005.

Schiller, Gertrud: Ikonographie der christlichen Kunst. Bd. 4,2. Maria. Gütersloh 1980.

Schreiner, Klaus: Maria, Jungfrau, Mutter, Herrscherin. München 1996 (Deutscher Taschenbuch Verlag 4707).

Schulze-Belli, Paola: Oswald's St Mary's Songs: Religious or Courtly Poetry? In: Jahrbuch der Oswald von Wolkenstein Gesellschaft 13 (2001/2002), S. 279-296.

Schiendorfer, Max: Ain schidlichs streuen. Heilsgeschichte und Jenseitsspekulation in Oswalds verkanntem Tagelied KI 40. In: Jahrbuch der Oswald von Wolkenstein-Gesellschaft 9 (1996/97), S. $179-196$.

Schwob, Anton, Ute Monika Schwob (Hgg.): Die Lebenszeugnisse Oswalds von Wolkenstein. 5 Bde. Wien 1999-2013.

Söll, Georg: Maria in der Geschichte von Theologie und Frömmigkeit. In: Handbuch der Marienkunde. Hg. von Wolfgang Beinert, Heinrich Petri. Regensburg 1984, S. 93-231.

Spicker, Johannes: Oswald von Wolkenstein. Die Lieder. Berlin 2007 (Klassiker-Lektüren 10), S. $96-102$.

Tervooren, Helmut: Minnesang, Maria und das „Hohe Lied“ - Bemerkungen zu einem vernachlässigten Thema. In: Vom Mittelalter zur Neuzeit. Festschrift für Horst Brunner. Hg. von Dorothea Klein zusammen mit Elisabeth Lienert und Johannes Rettelbach. Wiesbaden 2000, S. $15-47$.

Wachinger, Burghart: Blick durch die braw. Maria als Geliebte bei Oswald von Wolkenstein. In: Fragen der Liedinterpretation. Hg. von Hedda Ragotzky, Gisela Vollmann-Profe, Gerhard Wolf. Stuttgart 2001, S. 103-117.

Die Lieder Oswalds von Wolkenstein. Hg. von Karl Kurt Klein. 4., grundlegend neu bearb. Aufl. v. Burghart Wachinger. Berlin, Boston 2015 (Altdeutsche Textbibliothek 55). 
